\title{
Picosecond Relaxation of X-ray Excited GaAs
}

\author{
Victor Tkachenko* and Vladimir Lipp \\ Center for Free-Electron Laser Science CFEL, \\ Deutsches Elektronen-Synchrotron DESY, \\ Notkestrasse 85, 22607 Hamburg, Germany \\ Nikita Medvedev \\ Institute of Physics, Academy of Science of Czech Republic, \\ Na Slovance 1999/2, 18221 Prague 8, Czech Republic \\ Institute of Plasma Physics, Academy of Science of Czech Republic, \\ Za Slovankou 1782/3, 18200 Prague 8, Czech Republic and \\ Center for Free-Electron Laser Science CFEL, \\ Deutsches Elektronen-Synchrotron DESY, \\ Notkestrasse 85, 22607 Hamburg, Germany \\ Beata Ziaja \\ Center for Free-Electron Laser Science CFEL, \\ Deutsches Elektronen-Synchrotron DESY, \\ Notkestrasse 85, 22607 Hamburg, Germany and \\ Institute of Nuclear Physics, Polish Academy of Sciences, \\ Radzikowskiego 152, 31-342 Kraków, Poland
}

(Dated: May 15, 2017)

In this paper we present the current status of our theoretical studies on ultrafast relaxation of X-ray/XUV excited gallium arsenide. First, we discuss our previous approach, the unified model based on rate equations, two-temperature model and the extended Drude approach. By fitting the model to the available experimental data, we obtained realistic estimates on transient electronic temperature and electron-lattice thermalization timescale. Next, we make a step towards a rigorous

\footnotetext{
* Corresponding author:V. Tkachenko (victor.tkachenko@desy.de)
} 
description of the relaxation process with our hybrid code, XTANT. We extend the XTANT to include the band-specific effect of the suppression of collisional processes in GaAs, and perform dedicated simulations. We find that the extended model correctly describes the predicted transient non-isothermality of conduction and valence bands, however, currently, it cannot reproduce the experimentally observed reflectivity overshooting at $5-10$ ps. The reason for this discrepancy is that the electron-phonon coupling rate implemented in XTANT, although successfully applied for diamond and silicon, clearly underestimates the strength of this coupling in GaAs. The outline for a respective model improvement is discussed.

\section{INTRODUCTION}

Gallium arsenide is a semiconductor widely used for various industrial applications. It has a $1.42 \mathrm{eV}$-wide band gap (at room temperature [1, 2]) which is direct, i.e., the minimalenergy state in the conduction band $(\mathrm{CB})$ and the maximal-energy state in the valence band (VB) are characterized by the same crystal momentum (k-vector) in the Brillouin zone. Such band gap width is slightly smaller than the energy of the optical photons at $800 \mathrm{~nm}$ $\left(E_{\gamma}=1.55 \mathrm{eV}\right)$. If a small decrease in the band gap width occurs, it can trigger a noticeable increase in the (linear) absorption rate of optical photons. Thus, in addition to the free carrier absorption, incoming optical photons may also trigger an electron excitation from the valence to the conduction band, which then becomes the dominant channel of the optical energy absorption $[2,3]$. The rate of this interband excitation depends, apart from the band width, on the band occupations. As the band gap width in semiconductors is a function of the lattice temperature, the interband transition rate can reflect the transient changes of the lattice temperature. In this study we report on our theoretical studies of the relaxation of X-ray excited GaAs, utilizing transient optical properties of the sample which are sensitive to the interband transitions $[4-6]$.

Electronic excitation and relaxation processes following the FEL irradiation of solids are already quite well understood (see, e.g., [7-9]). The arriving FEL photons excite electrons from the valence to the conduction band and, at sufficiently high photon energies, also from the atomic inner shells. In light elements the inner-shell electron excitation is followed by an Auger decay, resulting in the emission of another electron into the conduction band. 
Energetic electrons within the conduction band can excite more secondary electrons from the valence band through electron impact ionization. The inverse process is Auger recombination [10] (i.e., three-body recombination) which reduces the density of the conduction band electrons. The electrons also interact with each other, exchanging energy. This leads to a fast thermalization of the conduction band electrons. Its timescale $\tau_{e l}$ depends on the FEL photon energy and fluence. At a few tens of femtoseconds corresponding to a typical FEL pulse duration, the electron thermalization timescale was reported to be $\leq 200$ fs both by theory $[7,8]$ and experiments $[11,12]$. Similar timescales were found in experiments with optical excitation [13-15]. A recent study [16] showed that the electron thermalization timescale is mainly determined by the secondary electron cascading following a FEL irradiation. The cascading duration increases with the increasing photon energy. Due to the ultrafast laser excitation, while electrons of the valence band are being transferred to the conduction band, holes are created in the valence band. The holes can be treated as quasi-particles with a positive charge and an effective mass [17]. The effective masses of holes and electrons in semiconductors depend on the specific band structure of the material. The dispersion relation at the bottom/top of a band can be approximated in many semiconductors as parabolic [18]:

$$
E(k)=E_{\text {edge }}+\hbar^{2} k^{2} / 2 m^{*},
$$

where $E(k)$ is the energy of an electron with a wave vector $\mathbf{k}$ in the band, $E_{\text {edge }}$ is a constant which stands for the value of the edge of the band, and $m^{*}$ is the effective mass. The effective mass is then directly connected to the curvature of the energy band at a specified k-vector, $\mathrm{k}_{0}$ :

$$
1 / m^{*}=d^{2} E(k) /\left.\hbar^{2}(d k)^{2}\right|_{k=k_{0}} .
$$

With a decrease of the second derivative of $E(k)$, the mass of the corresponding carrier increases. For example, if the top of the valence band is 'flatter' than the bottom of the conduction band, the effective hole mass exceeds the electron mass in the same material. In GaAs holes are about ten times heavier than electrons $[4,19]$. One can then expect that the absorbed energy (brought by X-ray photons) will be initially shared mostly among the light conduction band electrons [4] and only later transferred to the heavy holes. Such mutual (interband) thermalization between the valence and the conduction band may take significantly longer time than the carrier thermalization within the individual bands (intraband 
thermalizations). The interband thermalization is driven by collisional interband processes: collisional ionization and Auger recombination. Such transitions require both energy and wave vector conservation. Band structure has a strong effect on the rate of such processes [10]. Since GaAs is a direct band gap semiconductor, direct interband transitions between the valence and the bottom of the conduction band are suppressed as they cannot maintain both the conservation laws. Generally, phonon-assisted transitions give second-order contribution to collisional processes when compared to the first-order direct transitions [20]. I.e., they have much lower rates than the direct transitions [20], if the direct transitions are allowed. As the result, collisional interband transitions are strongly suppressed in the case of direct semiconductors with band gap widths larger than $\sim 1 \mathrm{eV}$ [10], in which fast direct interband transitions are not allowed and only slow phonon-assisted interband transitions can occur. Consequently, the distributions of carriers in the conduction and valence band remain for some time strongly non-isothermal. Similar carrier non-isothermality has been observed in Refs. [21, 22] and recently in [23].

In semiconductors, the timescale of the energy exchange between the free carriers and lattice, $\tau_{e l-l a t t}$ is of the order of picoseconds, which is typically much longer than the intraband electron thermalization time $[24,25]$. While the temperature of the lattice increases, the band gap shrinks. Based on the experimental data, phenomenological fits were constructed to describe the band gap shrinking as a function of the lattice temperature $[2,26-28]$. We consider here the case when the densities of excited carriers are too low to induce an additional band gap changes [29]. After the free carriers thermalize, recombination processes begin to affect the material evolution. In GaAs both radiative and non-radiative recombination occur [30]. However, the typical recombination times for GaAs are of the order of $\tau_{\text {rec }} \sim 100 \mathrm{~ns}$ [30], which is much longer than the electron-lattice thermalization time [24]. Fig. 1 shows the timescales of the predominant excitation and relaxation processes in X-ray irradiated GaAs.

Experimentally, these processes can be followed in a time resolved way by recording transient optical properties of the irradiated solid. A pump pulse from X-ray FEL initiates a process which is probed with an optical laser pulse, delayed by a certain time interval with respect to the pump pulse. It was already found during the first experiments on solid-state targets with FELs that an intense X-ray FEL laser pulse excites many electrons within the irradiated solid $[11,31]$. This leads to a transient change of the optical properties 
within the target which follow the evolution of the electron density. In particular, the measurements of transient optical properties allow to estimate the delay between pump and probe pulse. Therefore, in the first $[11,31]$ and later experiments [32-36] the transient optical properties of solids have been exploited to design non-destructive time-delay diagnostic and pulse characterization tools for FELs.

In addition, this experimental scheme also opens up possibilities to extract information about the relaxation processes within the laser-excited materials. Similar techniques have been used in two-color experiments with optical lasers to extract information on the electronphonon coupling coefficient [37] or to measure the temperature-dependent electron-lattice thermalization time $[24,25,38]$. Transient changes of transmission within X-ray irradiated GaAs were reported in Ref. [39] and interpreted as a signature of non-equilibrium relaxation effects within the irradiated solid. In [4], we identified the experimentally detected reflectivity overshooting as a signature of the electron-lattice thermalization due to electronphonon coupling and used this observation to determine the electron-lattice thermalization timescale. In [40] we explored this effect by a detailed modeling, which confirmed a nontrivial interplay between the electron-lattice coupling, band gap shrinkage, and the transient changes of optical properties.

In this work, we discuss our theoretical models to follow the relaxation of X-ray excited GaAs at the timescales up to a few tens of picoseconds. In Section II we describe the implementation of a simpler unified model based on the Drude approach, two-temperatureand rate equations which was applied in [4]. Afterwards, in Section III we analyze the applicability of a more rigorous model, XTANT [41] for GaAs, and discuss its possible improvements.

\section{ELECTRON-LATTICE THERMALIZATION IN GALLIUM ARSENIDE MODELLED WITH UNIFIED MODEL}

Our initially proposed theoretical framework for the interpretation of experimental data $[11,12]$ used rate equations to describe temporal evolution of the electron distribution [4]. The rate equations were coupled with the two-temperature model [42], describing the equilibration of the electron-lattice temperature. The Drude model was applied to follow the transient reflectivity as a function of free-carrier density. It was extended beyond the free- 
carrier absorption framework [26] to account for the contribution from optically induced interband transitions, predominant in this regime.

As a first step, we estimated the maximal electron-hole density after FEL irradiation, knowing the pulse fluence and the photoabsorption cross section at a given photon energy. We then used this value to reproduce the increase of the electron-hole density until the maximal density was reached. Such increase is typical for FEL irradiated semiconductors as described, e.g., in $[7,8]$. After the maximal electron-hole density was reached, the system started to relax. Electron-lattice thermalization and electron-hole recombination were the predominant relaxation channels. In GaAs, the latter one contributes much less significantly, as the typical recombination timescales are of the order of $100 \mathrm{~ns}$ [30]. Electron-lattice thermalization in GaAs acts on picosecond timescales [24, 25]. The resulting lattice heating should lead to a shrinkage of the band gap, $E_{\text {gap }}[27]$, when compared with its initial width of $1.42 \mathrm{eV}$ at $300 \mathrm{~K}$. This could result in the experimentally observed 'overshooting' of the transient reflectivity, i.e., its increase above the initial value. We confirmed this hypothesis by comparing model predictions to the available experimental data. Indeed, we identified the reflectivity overshooting as a result of the band gap shrinkage and obtained predictions for the electron-lattice thermalization time as a function of the pulse fluence and photon energy.

We proceeded in the following steps. The extended Drude model taking into account the interband contribution was used to calculate the transient reflectivity changes from the dielectric function, $\epsilon$, which is the function of optical coefficients, $n$ and $k$. The dielectric function is parametrized in the same way as in Ref. [26]:

$$
\epsilon \equiv(n+i k)^{2}=\epsilon_{\text {core }}-\sum_{j=e, h} \frac{\omega_{p, j}^{2}}{\omega_{\gamma}^{2}} \frac{\left(\omega_{\gamma} \tau_{j}\right)^{2}}{\left(\omega_{\gamma} \tau_{j}\right)^{2}+1}+\sum_{j=e, h} i \frac{\omega_{p, j}^{2}}{\omega_{\gamma}^{2}} \frac{\omega_{\gamma} \tau_{j}}{\left(\omega_{\gamma} \tau_{j}\right)^{2}+1},
$$

where $\epsilon_{\text {core }}$ describes all contributions to the dielectric function beyond the free-carrier absorption. Here, $\epsilon_{\text {core }}=\left(n_{\text {core }}+i k_{\text {interband }}\right)^{2}$, where $k_{\text {interband }}=\alpha \lambda_{\gamma} / 4 \pi$ describes the contribution from the transition between the valence and the conduction bands, using the interband absorption coefficient for a direct interband transition, $\alpha$, parametrized as in Ref. [26] (Eq. (5.31) therein). The frequency $\omega_{\gamma}$ is the photon frequency and $\lambda_{\gamma}$ is its corresponding wavelength. The absorption coefficient scales with the photon energy $E_{\text {phot }}$, as $\alpha \sim \sqrt{E_{\text {phot }}-E_{\text {gap }}} / E_{\text {phot }}$. Band gap shrinkage is described with the phenomenological relation from Ref. [27]. The interband absorption coefficient also contains the matrix element, 
$\langle v|p| c\rangle$, which couples the states with the same electron wave vector in the valence and the conduction bands. We parametrized it, using the measured absorption coefficients for GaAs from Ref. [3] (Fig. 3 therein). The time $\tau_{e(h)}$ is the electron (hole) collision time and the frequency $\omega_{p, e(h)}$ is the plasma frequency for electrons (holes). The latter one is estimated with the carrier density $n_{e-h}$ as $\omega_{p(e, h)} \sim \sqrt{n_{e-h}}$.

The average electron collision time $\tau_{e}$ was fitted in order to match the minimum of the experimental $\Delta R / R$ curve from Ref. [11, 12]. The accuracy of the fit depends on the resolution at which the minimum reflectivity was experimentally measured. The average hole collision time $\tau_{h}$ can be estimated from the electron one, using mass scaling relation for electron and hole collision frequencies [17]. The initial value of the lattice temperature is $300 \mathrm{~K}$. Lattice temperature does not change much during the first $100-200$ fs after the FEL irradiation (during the electron thermalization) and so the band gap width does not change either.

Rate equations describe changes of electron-hole density due to ionization and recombination processes. Heat capacities of the free-electron gas from Ref. [17] and of the lattice from Ref. [2] were used in the temperature equation, describing the exchange of thermal energy between free electrons and lattice. Fitted (i.e., iteratively adjusted) model parameters are: (i) the free-electron temperature at the minimum of $\Delta R / R$ curve, $T_{e}^{\text {init }}$, and (ii) the thermalization time, $\tau_{\text {el-latt }}$. Fig. 2 shows the resulting theoretical calculations (solid lines) compared to the experimental data (points).

The ultrafast drop and rise of the optical reflectivity occurs due to the production of electron-hole pairs in the irradiated semiconductors as described in Ref. [35]. The created free carriers alter the optical properties of the material. As we explained above, their contribution can be described by the Drude model.

The calculations shown in Fig. 2a were performed with the experimentally measured fluence values. The Drude model could not be applied at the fluence value, measured by Gahl et al., since such a fluence would lead to an overcritical electron density. However, at the pulse fluences close or larger than the one leading to the critical electron density, the minimum of $\Delta R / R$ curve was the same (Fig. 3b in [11]). Therefore, we could obtain the results presented in Fig. 2b, using a fluence value, corresponding to a subcritical electron density, at which the Drude model was still applicable. The predictions for $400 \mathrm{~nm}$ were obtained for the same FEL pulse parameters. Note that they cannot be directly compared 

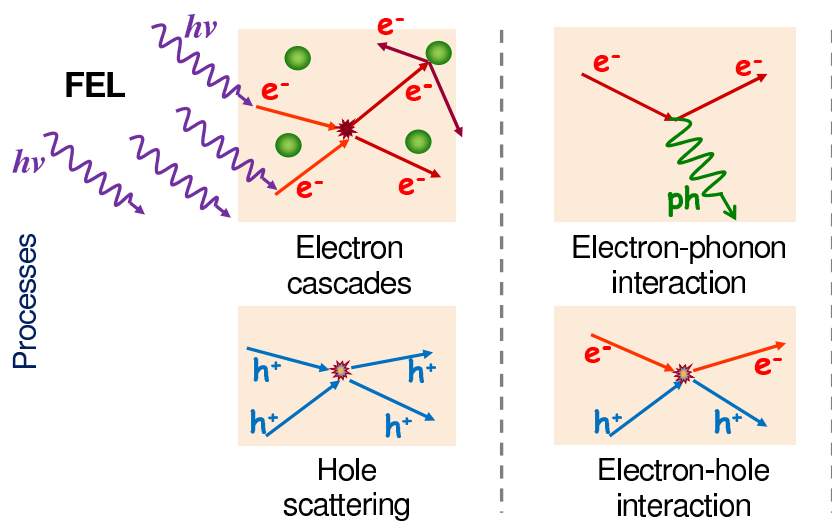

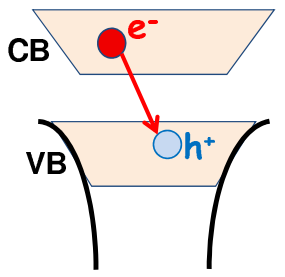

Electron-hole recombination

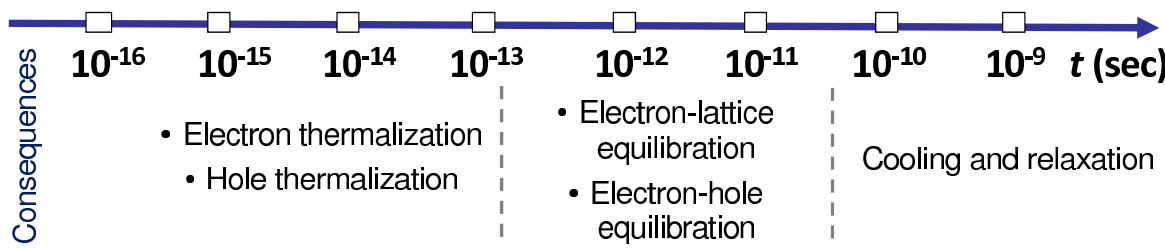

FIG. 1: (Color online) Timescales for predominant excitation and relaxation processes in X-ray irradiated GaAs. Figure reproduced from Ref. [4].

(a)

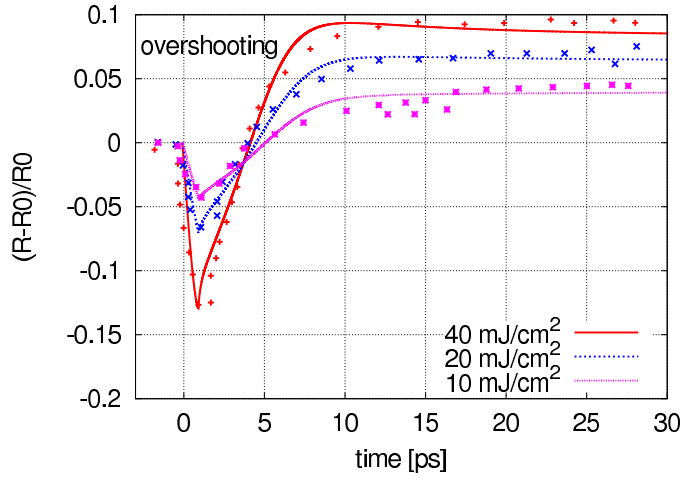

(b)

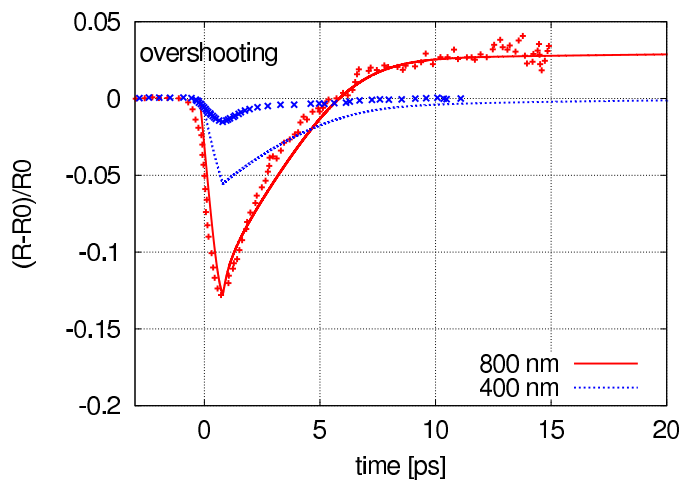

FIG. 2: (Color online) Relative change of transient reflectivity in GaAs as a function of time measured in: (a) the experiment by Krupin et al. [12] at FEL photon energy of 800 eV and optical probe of $800 \mathrm{~nm}$, (b) the experiment by Gahl et al. [11] at FEL photon energy of $40 \mathrm{eV}$. Experimental values (points) and theory predictions (lines) are compared for: (a) various FEL fluences: $F=10,20$, and $40 \mathrm{~mJ} / \mathrm{cm}^{2}$, and (b) various wavelengths of probe pulse: $800 \mathrm{~nm}$ and 400 nm. The theory results in (b) are obtained for a FEL pulse fluence of $F=4.1 \mathrm{~mJ} / \mathrm{cm}^{2}$. Figure reproduced from Ref. [4]. 
to the experimental curve from Fig. 2b in [11]: according to Fig. 3a therein, it corresponds to a different set of FEL pump parameters.

The optical coefficients are sensitive to the value of absorption coefficient which, according to various estimates, may differ by a factor of $2-3[2,3]$. This strongly influences the accuracy of the theory predictions (Fig. 2). At a $400 \mathrm{~nm}$ probe-pulse the interband absorption coefficient is about 100 times larger than at $800 \mathrm{~nm}[2,3]$. The reflectivity overshooting does not occur at $400 \mathrm{~nm}$ as the large absorption coefficient is then not sensitive to the small change caused by the energy shift due to the band gap shrinking $\left(\alpha \sim \sqrt{E_{\text {phot }}-E_{\text {gap }}} / E_{\text {phot }}\right)$.

Table I lists the parameters obtained through an iterative model adjustment to the experimental curves in Fig. 2a: the thermalization time, $\tau_{\text {el-latt }}$ and the free electron temperature at the minimum of $\Delta R / R$ curve, $T_{e}^{\text {init }}$. These values lay within the range reported in Ref. [24]. Note the expected increase of $T_{e}^{\text {init }}$ with fluence, and the corresponding decrease of the thermalization time with the electron temperature [24]. The values of $\tau_{\text {el-latt }}$ and $T_{e}^{\text {init }}$ for Fig. $2 \mathrm{~b}$ (experiment by Gahl et al.) at the pulse fluence of $F=4.1 \mathrm{~mJ} / \mathrm{cm}^{2}$ are 2.8 ps and $1.6 \mathrm{eV}$, respectively, for both $800 \mathrm{~nm}$ and $400 \mathrm{~nm}$.

\begin{tabular}{|c|c|c|}
\hline$F\left[\mathrm{~mJ} / \mathrm{cm}^{2}\right]$ & $\tau_{\text {el-latt }}[\mathrm{ps}]$ & $T_{e}^{\text {init }}[\mathrm{eV}]$ \\
\hline 40 & 2.0 & 2.8 \\
\hline 20 & 2.5 & 2.2 \\
\hline 10 & 3.0 & 1.6 \\
\hline
\end{tabular}

TABLE I: Model parameters iteratively adjusted to obtain predictions in Fig. 2a: thermalization time $\left(\tau_{\text {el-latt }}\right)$ and the free electron temperature at the minimum of $\Delta R / R$ curve $\left(T_{e}^{i n i t}\right)$. Table reproduced from Ref. [4]

\section{APPLICATION OF XTANT MODEL TO DESCRIBE RELAXATION IN X-RAY EXCITED GALLIUM ARSENIDE}

In what follows we use a more rigorous model, the in-house code XTANT (which stands for 'X-ray induced thermal and nonthermal transitions') [6, 41, 43-45] to trace the transient electron kinetics as well as atom dynamics. This hybrid model separates all electrons into high-energy and low-energy fraction. The high-energy electrons (with energies above $10 \mathrm{eV}$ ) 
are modeled with the Monte Carlo method, following propagation of individual particles and their collisions event-by-event. The low-energy electrons are traced with a temperature model including Boltzmann collision integral for the electron-ion energy exchange [41]. The electron-lattice interaction has been included into the simulation in the Fermi's Golden Rule (FGR) and in the so called 'dynamical coupling' frameworks (DC) [40]. Thus, the low-energy electrons are characterized by the Fermi distribution function, $f\left(E_{n}\right)$, evolving in time with the changes of the electron chemical potential and temperature. Atomic motion is tracked with Ehrenfest molecular dynamics technique (MD) and upgraded after each MD timestep, $\Delta t$ of duration up to $\sim 0.1 \mathrm{fs}$. The potential energy surface entering the Newtonian equations of motion for atoms is obtained from the transferable tight binding Hamiltonian. We choose orthogonal transferable tight binding models, which can interpolate the properties of the band-structure between different phases of a material, i.e., at a changing atomic geometry. Here we use the specific tight binding parametrization for GaAs from Ref. [46]. Due to the limitations of the minimal basis set, such tight binding models can only satisfactorily describe band structure of the valence band and of the bottom of the conduction band. This affects the accuracy of complex dielectric function (CDF) calculated at higher photon energies, when a certain number of electrons is excited to high-lying states in the conduction band [6]. Here, we use the calculated CDF and the resulting optical parameters only within low energy regime in which they are reliable.

An extension of XTANT to enable an on-the-fly description of transient optical properties was proposed in [5] and successfully verified by comparison to the available data on optically excited diamond and silicon. In [6] the code has been for the first time applied to describe time evolution of X-ray excited GaAs. Fig. 3 shows the predictions obtained for GaAs at the absorbed dose of $\sim 0.2 \mathrm{eV} /$ atom. This dose is much higher than the highest absorbed dose in the experiments by Gahl and Krupin $(\sim 0.08 \mathrm{eV} /$ atom $)$. Yet, the timescale of the reflectivity overshooting $\sim 15 \mathrm{ps}$ is much larger than that one observed in both experiments at the highest dose. In addition, the simulation results on the electron temperature are in disagreement with the results of the unified model from Ref. [4] described in Section II. According to the XTANT predictions, the electron temperature at the end of cascading, i.e,. at the minimum of the reflectivity curve is $\sim 0.4 \mathrm{eV}$, whereas the one predicted with the Drude model is $2-3 \mathrm{eV}$. The latter estimate of the electronic temperature was obtained from the average kinetic energy of a free electron within the conduction band, $E_{\text {el }}$ after 
neglecting the energy of the (heavy) hole with the relation:

$$
E_{\mathrm{el}}=E_{\mathrm{eh}}-E_{\text {gap }},
$$

where $E_{\text {eh }}$ is the average pair creation energy and $E_{\text {gap }}$ is the band gap width. In GaAs $E_{\text {gap }}$ $=1.42 \mathrm{eV}$ and $E_{\mathrm{eh}}=4.2 \mathrm{eV}$ [47], which gives a universal value of $E_{\mathrm{el}}=2.77 \mathrm{eV}$. From the relation $E_{\mathrm{el}}=3 / 2 k_{B} T_{\mathrm{el}}$, it follows that $T_{\mathrm{el}}=1.85 \mathrm{eV}$.

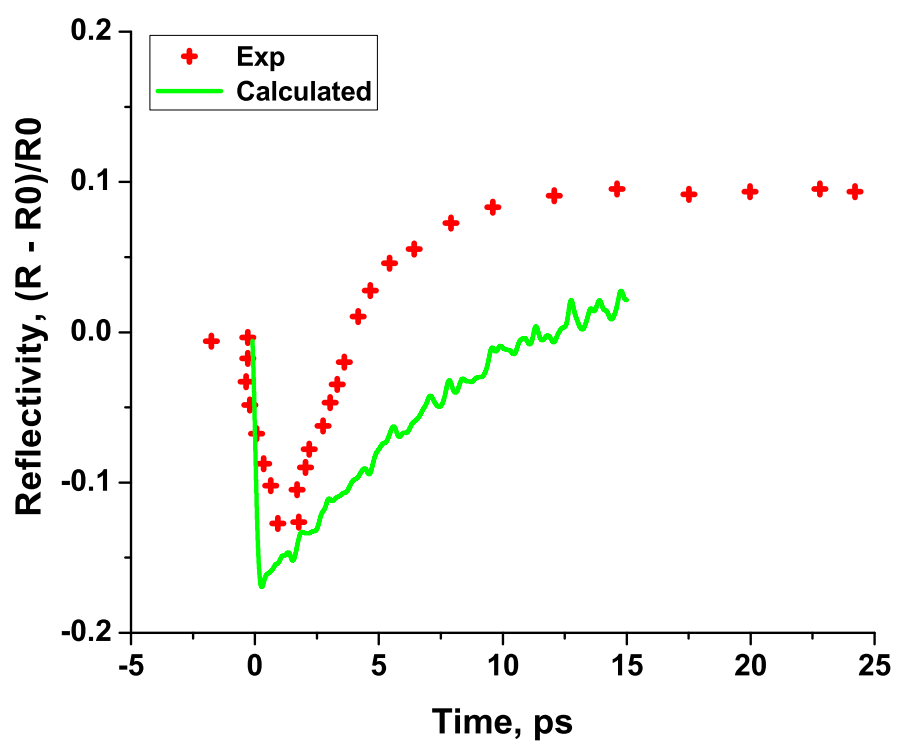

FIG. 3: Transient reflectivity of X-ray irradiated GaAs sample probed at $800 \mathrm{~nm}$ as simulated by XTANT (solid green line) at photon energy of $800 \mathrm{eV}$ and the absorbed dose $=0.2 \mathrm{eV} /$ atom; and measured experimentally in [12] (red points). Figure reproduced from Ref. [6].

The disagreement of the electronic temperatures can be explained by recalling the specific band structure of GaAs. The direct band gap leads to the supression of collisional interband processes as discussed above. However, the XTANT model intrinsically assumes an instantaneous mutual thermalization of electrons both in the conduction and in the valence band. The entire electronic system in the low energy domain is then represented by a single Fermi-Dirac distribution with a global chemical potential and a global electron temperature which are updated with the simulation progress at each time step [41]. This assumption does not hold in case of GaAs. In this case the model has to be extended in order to account for the suppressed collisional exchange between the bands. 
The simplest approach to account for delayed interband thermalization is to equilibrate the bands separately by assuming an independent thermalization of electrons in the conduction band with a specific electronic temperature, $T_{c}$ and the chemical potential, $\mu_{c}$ and in the valence band with the temperature, $T_{v}$ and chemical potential, $\mu_{v}$ respectively. A similar approach was already introduced, e.g., for Si by van Driel in [48]. Electrons in the conduction band and holes in the valence band were described there with separate FermiDirac distributions of different chemical potentials, $\mu_{e}$ and $\mu_{h}$, correspondingly, but with a global temperature, $T_{e}[48,49]$.

In order to separate interband thermalization in the framework of the XTANT model, we use a scheme analogous to the case of Fermi-Dirac distribution with a global chemical potential and a global electron temperature. At the first time step (before irradiation) we assume that the sample is in equilibrium, and calculate a global distribution function from the initial input parameters: total energy and carrier number. Later, we follow separate Fermi-Dirac distributions for each band. Namely, we calculate Fermi distribution function for electrons in each band by solving an inverse problem for a given number and energy of carriers within each band, analogously, as in Ref. [41]:

$$
\begin{gathered}
N_{v}^{\text {low }}=\sum_{E_{v, \text { min }}}^{E_{v, \text { max }}} f_{v}\left(E_{i}\right)=\sum_{E_{v, \text { min }}}^{E_{v, \text { max }}} \frac{2}{1+\exp \left(\left(E_{i}-\mu_{v}\right) / T_{v}\right)}, \\
N_{c}^{\text {low }}=\sum_{E_{c, \text { min }}}^{E_{c u t}} f_{c}\left(E_{i}\right)=\sum_{E_{c, \text { min }}}^{E_{c u t}} \frac{2}{1+\exp \left(\left(E_{i}-\mu_{c}\right) / T_{c}\right)}, \\
E_{v}^{\text {low }}=\sum_{E_{v, \text { min }}}^{E_{v, \text { max }}} E_{i} f_{v}\left(E_{i}\right)=\sum_{E_{v, \text { min }}}^{E_{v, \text { max }}} \frac{2 E_{i}}{1+\exp \left(\left(E_{i}-\mu_{v}\right) / T_{v}\right)}, \\
E_{c}^{\text {low }}=\sum_{E_{c, \text { min }}}^{E_{c u t}} E_{i} f_{c}\left(E_{i}\right)=\sum_{E_{c, \text { min }}}^{E_{c u t}} \frac{2 E_{i}}{1+\exp \left(\left(E_{i}-\mu_{c}\right) / T_{c}\right)} .
\end{gathered}
$$

where $E_{v, \min }$ is the energy at the bottom of the valence band, $E_{v, \max }$ is the energy at the top of the valence band, $E_{c, \min }$ is the energy at the bottom of the conduction band, and $E_{\text {cut }}$ is the high-energy cut-off in the conduction band $(\sim 10 \mathrm{eV})$. At each time step the XTANT code updates the information on the number of carriers and their energy in both bands. Using them, we calculate with the bisection method applied for each band the values of $T_{c}$ and the chemical potential, $\mu_{c}$, and, in the valence band, with the temperature, $T_{v}$ and the chemical potential, $\mu_{v}$ respectively at each time step. With the obtained distribution 
functions, we determine the global potential energy surface and then initiate a molecular dynamics calculation timestep. The energy incoming with the laser pulse is redistributed between the high-energy electrons and the low-energy domain. In the case of an inelastic scattering and energy loss by a high-energy electron (an impact-ionized, photoionized, or Auger one), we trace whether the electron remains in the high-energy domain or it falls either to the low-energy part of the conduction band or to the valence band. In this way, we trace the changes of carrier numbers and their energy in each band independently. Interband transition are then naturally suppressed within the low-energy domain. Fig. 4 shows the evolution of electron temperatures in valence and conduction bands simulated at the conditions of Krupin's and Gahl's experiments [11, 12]. Similarly to the case of the global Fermi distribution model, after the maximum of the pulse, a sharp increase in the valence and the conduction band electron temperatures is observed. The calculated peak value of the CB electron temperature $T_{c}$ exceeds $2 \mathrm{eV}$ both in simulations of Krupin's and Gahl's experiments (Fig. 4) which is close to the value $1.85 \mathrm{eV}$ estimated from Eq. (4). At the same time, the peak value of the $\mathrm{VB}$ electron temperature $T_{v}$ is around $0.4 \mathrm{eV}$. As expected, it is close to the global electron temperature $T_{e}=0.42-0.43$ calculated for the same simulation parameters, as there are many more electrons in the valence band than in the conduction band, and they provide the predominant contribution into the global electron temperature. The peak number of the created electron-hole pairs $n_{e-h}$ per atom exceeds $2 \%$ which is also in agreement with the value of $n_{e-h}$ estimated with the unified model from section II (about 2.2\%). This confirms that the extended XTANT model provides reliable results on the electron density and temperature. The code can then be further utilized to study the optical properties of X-ray excited GaAs. While performing the simulations, we included the electron-ion coupling in the way described in Refs. [6, 40]. According to our earlier finding [40], the dynamical electron-ion coupling scheme starts to converge at the MD time step $\Delta t \sim 0.01-0.02$ fs , whereas the Fermi's Golden Rule scheme is inherently divergent in this regime (i.e., for any time step chosen). Here, we show the predictions obtained with Fermi's Golden Rule scheme for purely illustrative purpose.

From the predictions shown in Fig. 4 for the MD timestep, $\Delta t=0.02 \mathrm{fs}$, we can see that during the electron-lattice thermalization process the $\mathrm{CB}$ electron temperature $T_{c}$ decreases significantly on a timescale of $10-15 \mathrm{ps}$, in contrast to the VB electron temperature $T_{v}$ which decreases very slowly. At the same time, the atomic temperature $T_{a}$ rises from the 

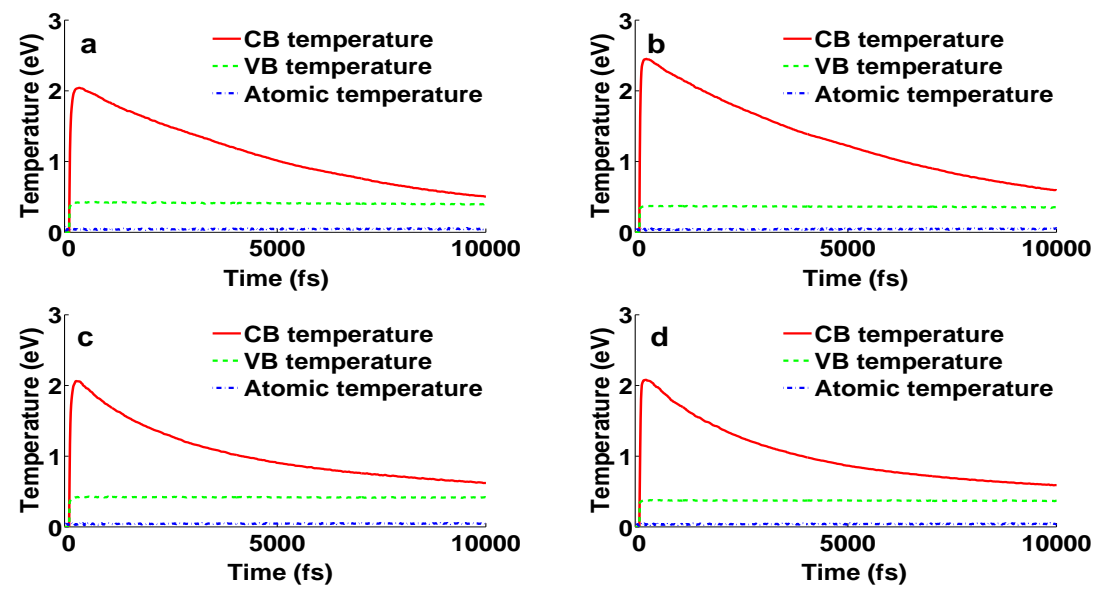

FIG. 4: Transient temperatures within X-ray excited GaAs: atomic temperature (dashed blue line), transient conduction band electron temperature (solid red line), and valence band electron temperature (dot-dashed green line). They were obtained with the XTANT simulations at the MD time step, $\Delta t=\mathbf{0 . 0 2}$ fs for: (a) Gahl's experiment, dynamical coupling; (b) Krupin's experiment, dynamical coupling; (c) Gahl's experiment, FGR coupling; (d) Krupin's experiment, FGR coupling. The pulse fluence was: $F=4.1 \mathrm{~mJ} / \mathrm{cm}^{2}$ for Gahl's experiment, $F=40 \mathrm{~mJ} / \mathrm{cm}^{2}$ for Krupin's experiment.

initial value of $300 \mathrm{~K}$ only up to $600-700 \mathrm{~K}$. As the result, the experimentally observed overshooting of reflectivity is not achieved even within 10 ps (Fig. 6). However, knowing the energy absorbed from the pulse, we can predict a final atomic temperature that the system would reach after the accomplished electron-lattice equilibration. In our case it would be $919 \mathrm{~K}$ for the conditions of Gahl's experiment and Krupin's experiment. The predicted final value of the reflectivity change, $\Delta R / R$ is $\sim 0.08$ - in good agreement with Krupin's experiment. In Gahl's experiment performed at lower photon energy, the experimental results are affected by carrier diffusion, therefore, the agreement between the diffusion-free simulation results and the measurement is worse than for Krupin's experiment.

These findings indicate that the proposed dynamical scheme for electron-phonon coupling, although successful for silicon and diamond, is not reliable in case of GaAs. As a result, our model underestimates the electron-ion coupling rate in GaAs. The reason for that might be the specific band structure of this material with a direct band gap. A possible improvement would require to go beyond the $\Gamma$ point approximation and to include multiple $k$-points in 

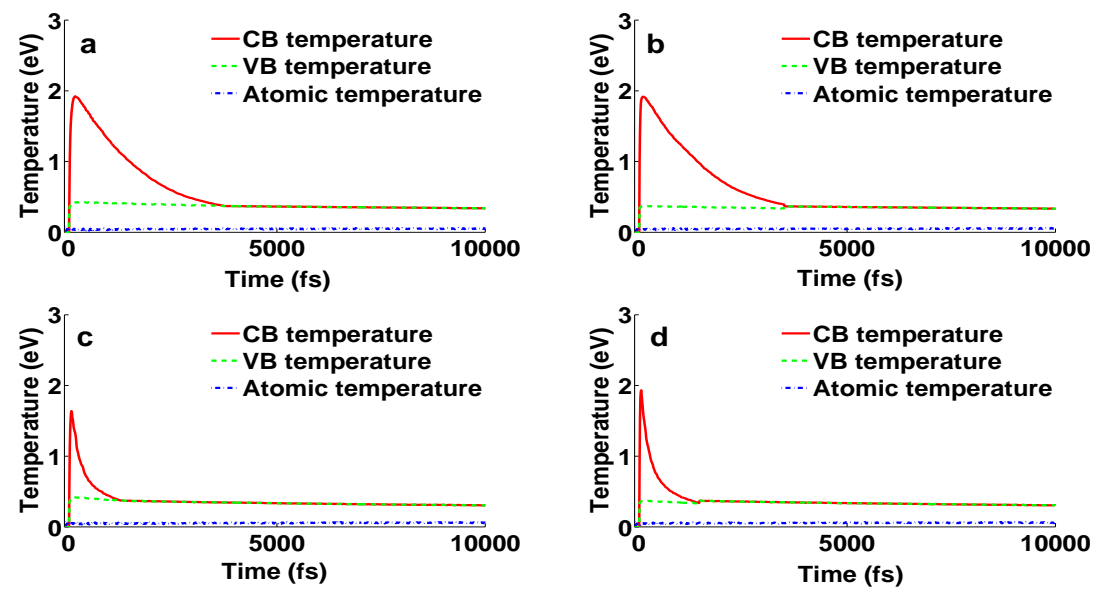

FIG. 5: Transient temperatures within X-ray excited GaAs: transient atomic temperature (dashed blue line), conduction band electron temperature (solid red line), and valence band electron temperature (dot-dashed green line). They were obtained with the XTANT simulations at the MD time step, $\Delta t=\mathbf{0 . 1}$ fs for: a) Gahl's experiment ( $\hbar \omega=40 \mathrm{eV})$, dynamical coupling; b) Krupin's experiment ( $\hbar \omega=800 \mathrm{eV})$, dynamical coupling; c) Gahl's experiment, FGR coupling; d) Krupin's experiment, FGR coupling. The pulse fluence was: $F=4.1 \mathrm{~mJ} / \mathrm{cm}^{2}$ for Gahl's experiment, $F=40 \mathrm{~mJ} / \mathrm{cm}^{2}$ for Krupin's experiment.

the evaluation of the coupling as it was performed, e.g., in [50]. However, this task is beyond the scope of the current paper.

To illustrate that the electron-lattice equilibration and the reflectivity overshooting are principally achievable in the framework of the current model under the condition of a correct electron-phonon coupling treatment, we performed simulations with the larger MD time step $\Delta t=0.1$ fs (Fig. 5) which artifically increases the rate of electron-ion coupling. As we know from our previous studies, this time step is sufficient for MD convergence but too large for the convergence of electron-ion coupling rate. Its value then is larger than the converged one. This artificial increase of the coupling rate shortens the interband thermalization timescale.

After the equilibration of temperature and chemical potential in both bands, we again use the global Fermi distribution, assuming, that the interband equilibration already occurred. Since the collisional electron transitions between the bands are then again permitted, the number of electrons in the conduction band starts to decrease. Its impact on the reflectivity coefficient can be seen in Fig. 6. Although the experimentally observed overshooting, 


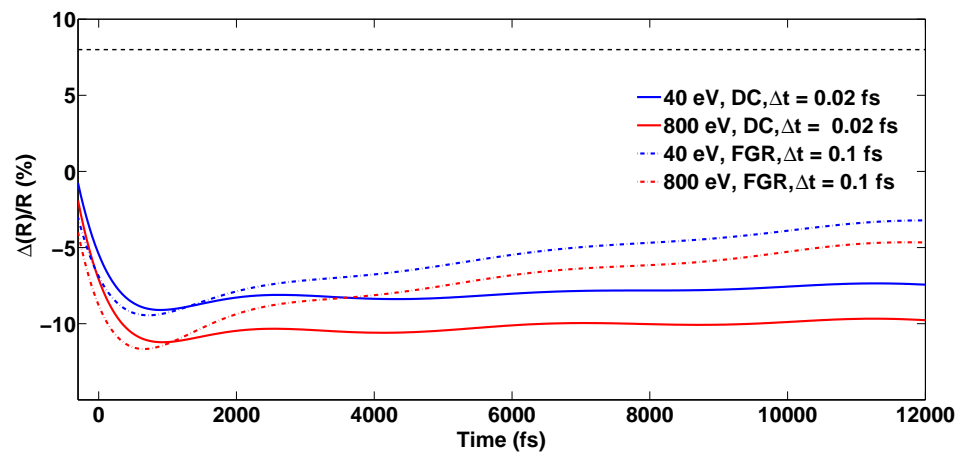

FIG. 6: Time dependence of the reflectivity change simulated with DC approach (solid lines) at $\Delta t=0.02 \mathrm{fs}$ and with FGR approach (dot-dashed lines) at $\Delta t=0.1 \mathrm{fs}$ both for Gahl's experiment ( $\hbar \omega=40 \mathrm{eV}$, blue lines) and Krupin's experiment ( $\hbar \omega=800 \mathrm{eV}$, red lines). The pulse fluence was: (i) $F=4.1 \mathrm{~mJ} / \mathrm{cm}^{2}$ for Gahl's experiment, and (ii) $F=40 \mathrm{~mJ} / \mathrm{cm}^{2}$ for Krupin's experiment. The black dashed line denotes the reflectivity change, which would be observed after the accomplished electron-lattice equilibration.

starting after $\sim 5$ ps and saturating at $\sim 10 \mathrm{ps,} \mathrm{still} \mathrm{could} \mathrm{not} \mathrm{be} \mathrm{exactly} \mathrm{reproduced,} \mathrm{due}$ to the too low electron-lattice coupling rate, the slope of the reflectivity is steeper after the artificial 'increase' of the coupling rate, when compared to the case of the MD time step $\Delta t=0.02$ fs within DC approach. This, again, confirms our expectation that the current electron-coupling model underestimates the electron-ion coupling rate in GaAs and requires a dedicated improvement.

To sum up, here we extended our XTANT simulation tool in order to account for the specific properties of GaAs. With the model, we reproduced correctly the initial nonisothermality of the valence and the conduction bands in X-ray irradiated GaAs. Furthermore, we identified the reason for the disagreeement between our predictions and the experimental data at picosecond timescales which is due to a too low electron-ion coupling rate currently implemented. After the necessary improvement of the electron-phonon coupling rate, we expect that the extended XTANT model should be able to describe quantitatively the complex relaxation of both soft and hard X-ray excited GaAs. 


\section{ACKNOWLEDGEMENTS}

Authors thank Martin Beye, Theophilos Maltezopoulos, Oriol Vendrell and Wilfried Wurth for helpful discussions.

\section{REFERENCES}

[1] Kittel C. Introduction to Solid State Physics, 6th Ed. New York:John Wiley, 1986.

[2] Siklitsky, V., Ioffe Physico-Technical Institute. Database on Semiconductors. http://www.ioffe.rssi.ru/SVA/NSM/Semicond/ (Date of access: 01/10/2014), 1998.

[3] Sturge, M. D. Optical Absorption of Gallium Arsenide between 0.6 and $2.75 \mathrm{eV}$. Phys. Rev., $127: 768,1962$.

[4] Ziaja B. et al. Time-resolved observation of band-gap shrinking and electron-lattice thermalization within X-ray excited gallium arsenide. Sci. Rep., 5:18068, 2015.

[5] Tkachenko V. et al. Transient optical properties of semiconductors under femtosecond x-ray irradiation. Phys. Rev. B, 93:144101, 2016.

[6] V. Tkachenko, N. Medvedev, and B. Ziaja. Transient changes of optical properties in semiconductors in response to femtosecond laser pulses. Appl. Sci., 6(9):238, 2016.

[7] Ziaja, B., van der Spoel, D., Szöke, A., and Hajdu, J. Auger-electron cascades in diamond and amorphous carbon. Phys. Rev. B, 64:214104, 2001.

[8] Medvedev, N., and Rethfeld, B. Transient dynamics of the electronic subsystem of semiconductors irradiated with an ultrashort vacuum ultraviolet laser pulse. New J. Phys., 12:073037073043, 2010.

[9] Fitting, H. J., Kortov, V. S., and Petite, G. Ultra-fast relaxation of electrons in wide-gap dielectrics. J. of Lum., 122:542, 2007.

[10] Haug A. Auger recombination in direct-gap semiconductors: band-structure effects. J. Phys.C, 16:4159, 1983.

[11] Gahl, C. et al. A femtosecond X-ray/optical cross-correlator. Nat. Phot., 2:165, 2008.

[12] Krupin, O. et al. Temporal cross-correlation of x-ray free electron and optical lasers using soft 
x-ray pulse induced transient reflectivity. Opt. Exp., 20:11396, 2012.

[13] Knox, W. H. et al. Femtosecond excitation of nonthermal carrier populations in GaAs Quantum Wells. Phys. Rev. Lett., 56:1191, 1986.

[14] Oudar, J. L. et al. Femtosecond orientational relaxation of photoexcited carriers in GaAs. Phys. Rev. Lett., 53:384, 1984.

[15] Kim, A. M. T. et al. Dynamics and phase changes in crystalline and amorphous GaAs. Phys. Rev. B, 66:245203, 2002.

[16] Medvedev, N. et al. Electron kinetics in femtosecond X-Ray irradiated SiO2. Contr. Plasma Phys., 53:347, 2013.

[17] Ashcroft, N.W., and Mermin, N.D. Solid state physics. Harcourt, Inc., 1976.

[18] Grosso G. and Pastori Parravicini G. Solid State Physics. San Diego: Academic Press, 2000.

[19] Blatt F.J. Modern Physics. McGraw-Hill, 1992.

[20] Sano N. and Yoshii A. Impact-ionization theory consistent with a realistic band structure of silicon. Phys. Rev. B, 45:4171, 1992.

[21] Joshi, R. P., Grondin, R. O., and Ferry, D. K. Monte Carlo simulation of electron-hole thermalization in photoexcited bulk semiconductors. Phys. Rev. B, 42:5685, 1990.

[22] Tomassi, R., Langot, P., and Vallee, F. Femtosecond hole thermalization in bulk GaAs. Appl. Phys. Lett., 66:1361, 1995.

[23] Hajlaoui, M. et al. Tuninig a Schottky barrier in a photoexcited topological insulator with transient Dirac cone electron-hole asymmetry. Nat. Commun., 5:3003, 2014.

[24] Del Fatti, N. et al. Temperature-dependent electron-lattice thermalization in GaAs. Phys. Rev. B, 59:4576, 1999.

[25] Langot, P. et al. Femtosecond investigation of the hot-phonon effect in GaAs at room temperature. Phys. Rev. B, 54:14487, 1996.

[26] Dresselhaus, M. S. Solid state physics II: Optical Properties of Solids. http://web.mit.edu/course/6/6.732/www/6.732-pt2.pdf, 2017.

[27] Blakemore, J. S. Semiconducting and other major properties of gallium arsenide. J. Appl. Phys., 53:R123, 1982.

[28] Beye M. et al. Measurement of the predicted asymmetric closing behaviour of the band gap of silicon using x-ray absorption and emission spectroscopy. New J. Phys., 12:043011, 2010.

[29] Spataru, C. D., Benedict, L. X., and Louie. S. G. Ab initio calculation of band-gap renormal- 
ization in highly excited GaAs. Phys. Rev. B, 69:205204, 2004.

[30] Saleh, B. E. A., and Teich, M. C. Fundamentals of Photonics. J.Wiley and Sons, ISBN: 0-471-83965-5, 1991.

[31] Maltezopoulos, T. et al. Single-shot timing measurement of extreme-ultraviolet free-electron laser pulses. New J. Phys., 10:033026, 2008.

[32] Krikunova, M. et al. Time-resolved ion spectrometry on xenon with the jitter-compensated soft x-ray pulses of a free-electron laser. New J. Phys., 11:123019, 2009.

[33] Krikunova, M. et al. Ultrafast photofragmentation dynamics of molecular iodine driven with timed XUV and near-infrared light pulses. J. Chem. Phys., 134:024313, 2011.

[34] Krikunova, M. et al. Strong-field ionization of molecular iodine traced with XUV pulses from a free-electron laser. Phys. Rev. A, 86:043430, 2012.

[35] Harmand, M. et al. Achieving few-femtosecond time-sorting at hard X-ray free-electron. Nat. Phot., 7:215, 2013.

[36] Riedel, R. et al. Single-shot pulse duration monitor for extreme ultraviolet and X-ray freeelectron lasers. Nat. Commun., 4:1731, 2013.

[37] Hostetler, J. L. et al. Measurement of the electron-phonon coupling factor dependence on film thickness and grain size in Au, Cr, and Al. Appl. Opt., 38:3614, 1999.

[38] Cho, G. C., Kuett, W., and Kurz, H. Subpicosecond time-resolved coherenbt-phonon oscillations in GaAs. Phys. Rev. Lett., 65:764, 1990.

[39] Durbin, S. M. et al. X-ray pump optical probe cross-correlation study of GaAs. Nat. Phot., $6: 111,2012$.

[40] N. Medvedev, Z. Li, V. Tkachenko, and B. Ziaja. Electron-ion coupling in semiconductors beyond Fermi's golden rule. Phys. Rev. B, 95:014309, 2017.

[41] Medvedev N., Jeschke H. O., and Ziaja B. Nonthermal phase transitions in semiconductors induced by a femtosecond extreme ultraviolet laser pulse. New J. Phys., 15:015016, 2013.

[42] Anisimov, S. I., Kapeliovich, B. L., and Perelman, T. L. Electron emission from metal surfaces exposed to ultrashort laser pulses. Sov. Phys. JETP, 39:375, 1974.

[43] Medvedev N., Tkachenko V., and Ziaja B. Modeling of nonthermal solid-to-solid phase transition in diamond irradiated with femtosecond x-ray FEL pulse. Contrib. Plasma Phys., 55:12, 2015.

[44] Medvedev N., Jeschke H.O., and Ziaja B. Nonthermal graphitization of diamond induced by 
a femtosecond x-ray laser pulse. Phys. Rev. B, 88:224304, 2013.

[45] N. Medvedev, Z. Li, and B. Ziaja. Thermal and nonthermal melting of silicon under femtosecond x-ray irradiation. Phys. Rev. B, 91:054113, 2015.

[46] Molteni C., Colombo L., and Miglio L. Tight-binding molecular dynamics in liquid ii-v compounds: I. potential generation. J. Phys.: Condens. Matter, 6:5243, 1994.

[47] Siklitsky V. Ioffe Physico-Technical Institute. Database on Semiconductors http : //www.ioffe.rssi.ru/sva/nsm/semicond. 1998.

[48] van Driel H.M. Kinetics of high-density plasmas generated in Si by 1.06- and $0.53-\mu \mathrm{m}$ picosecond laser pulses. Phys. Rev. B, 35:8166, 1987.

[49] Lipp V. Atomistic-continuum modeling of ultrafast laser-induced melting of silicon targets. PhD thesis, University of Kassel, 2015.

[50] Liu T.H. et al. First-principles mode-by-mode analysis for electron-phonon scattering channels and mean free path spectra in GaAs. arXiv: 1606.07074, 2016. 